\title{
Risk of suicide in households threatened with eviction: the role of banks and social support
}

Inmaculada Mateo-Rodríguez ${ }^{1,2,3}$, Laura Miccoli ${ }^{1}$, Antonio Daponte-Codina ${ }^{1,2,4,6^{*}} \mathbb{D}$, Julia Bolívar-Muñoz ${ }^{1,2^{\wedge}}$, Cecilia Escudero-Espinosa ${ }^{2}$, M. Carmen Fernández-Santaella ${ }^{5}$, Jaime Vila-Castellar ${ }^{5}$, Humbelina Robles-Ortega ${ }^{5}$, José Luis Mata-Martín ${ }^{5}$ and Mariola Bernal-Solano ${ }^{1,2}$

\begin{abstract}
Background: One of the greatest effects of the financial crisis in Spain has been the enormous increase in the number of evictions. Several studies have shown the association of evictions with different aspects of the physical and mental health. Furthermore, evictions have been associated with an increased risk of suicide. Our objective was to evaluate the risk of suicide among victims of eviction and investigate whether it is associated with specific characteristics of households and interviewees, the eviction process and social support, and health needs.
\end{abstract}

Methods: A total of 205 participants from households threatened with eviction in Granada, Spain, and 673 being the total number of members of these households, were interviewed in one-on-one sessions between April 2013 and May 2014. Through a questionnaire, information was obtained on physical and mental health, characteristics of their eviction process and support networks, and the use of health services.

Results: Almost half of the sample (46.7\%) were at low (11.8\%), moderate (16.9\%), or high suicide risk (17.9\%). Household and interviewee features had a limited association with suicide risk. On the contrary, the risk of suicide is greater with a longer exposure to the eviction process. In addition, threatening phone calls from banks increased significantly the risk of suicide, especially among men. Suicide risk was also associated with low social support, especially among women. Interviewees at risk of suicide received more help from nongovernmental organizations than those who were not at risk. In interviewees at risk, the main unmet needs were emotional and psychological help, especially in men. A high percentage of those at risk of suicide declare having large unmeet health needs. Finally, there was a tendency among the evicted at risk of suicide to visit emergency room and primary care more often than those not at risk, especially among women.

Conclusions: To our knowledge, this is the first study showing that when banks adopt a threatening attitude, suicide risk increases among the evicted. As hypothesized, when the evicted felt socially supported, suicide risk decreased. Emotional help was the main mediator of suicide risk and the main unmet need, especially among men.

Keywords: Evictions, Foreclosure, Suicide risk, Social support, Banks

\footnotetext{
* Correspondence: antonio.daponte.easp@juntadeandalucia.es

Julia Bolívar-Muñoz is deceased. This paper is dedicated to her memory.

${ }^{1}$ CIBER Epidemiology and Public Health (CIBERESP), Instituto de Salud Carlos

III, Madrid, Spain

${ }^{2}$ Andalusian School of Public Health (EASP), Granada, Spain

Full list of author information is available at the end of the article
}

(c) The Author(s). 2019 Open Access This article is distributed under the terms of the Creative Commons Attribution 4.0 International License (http://creativecommons.org/licenses/by/4.0/), which permits unrestricted use, distribution, and reproduction in any medium, provided you give appropriate credit to the original author(s) and the source, provide a link to the Creative Commons license, and indicate if changes were made. The Creative Commons Public Domain Dedication waiver (http://creativecommons.org/publicdomain/zero/1.0/) applies to the data made available in this article, unless otherwise stated. 


\section{Backgroud}

In 2007, after decades of irresponsible mortgage practices [1], the financial sector suddenly crumbled, causing the worldwide collapse of the housing market and, in turn, an unprecedented increase in home foreclosures. The impact of the crisis was heterogeneous and in Spain, it hit Mediterranean regions such as Andalusia the most [2]. As in earlier economic crises, the Great Recession resulted in worse mental health and higher suicide rates [3-13], especially in low-income, high-unemployment areas [1, 7, 10, 14-16].

Home evictions consist of a long process, from mortgage arrears to the loss of the home, during which the household is increasingly pressured to pay back the loans. In Spain during the harshest years of the crisis (2008-2013) housing-related legal actions were essentially dispossessions related to home ownership rather than renting [17] . The eviction process has been associated with stress, loss of identity, shame, and failure [18-21], which lead to poor mental health $[7,16]$, visible in higher rates of depression and anxiety among the evicted, and symptomatology of post-traumatic stress disorder [PTSD] [21-23].

Suicide is the worst possible outcome of disturbed mental health [24]. It has been estimated that each suicide corresponds to about 20 failed attempts [25, 26]. Studies in several countries have consistently shown that home evictions are associated with higher suicide rates, even after adjusting for unemployment, debts, and previous mental health [7, 27-29]. In most countries, the evicted population comprises socially vulnerable groups, hence, it is hard to disentangle the impact of eviction from the impact of other forms of social marginalization. However, the main cause of evictions in Spain during the economic crisis was the loss of employment, mostly affecting the working middle class, unlike in other countries, where evictions are part of a complex process of marginalization of the structurally vulnerable population [15, 22, 28, 30].

Research on economic downturns and mental health across different countries emphasizes the role of the state [31]: states that adopted drastic measures of austerity by limiting welfare, such as Spain, Portugal, and Greece, reported sharper increases in mortality and suicide rates [10, 16, 32-34], as compared to countries that rejected austerity, such as Iceland, or provided formal support by increasing social welfare, such as Sweden and Finland- $[3,34,35]$. Overall, these findings highlight the mediating role of formal support in the relationship between economic downturns and suicides.

Spain is among the European countries hardest hit by the crisis [36]. In recent decades, government policies promoted housing as a central component of the Spanish economy, while eliminating key policies of employment protection, causing a sustained increase in low wages, insecurity and job insecurity [37, 38]. The housing market crash tripled national unemployment rates, which were the highest or the second-highest in Europe-28 from 2009 to 2018 [39], and struck Spain's working middle class [37], causing shattering levels of family poverty [40]. Furthermore, Spanish mortgage laws, criticized by 46 senior Spanish judges and denounced by the Court of Justice of the European Union [CJEU] [41], in case of arrears, do not offer alternatives to foreclosure and do not allow the mortgagor to satisfy the debt by selling/returning the property, therefore, the value of the property must still be paid after eviction. Consequently, a portion of Spain's middle class became part of the new poor and increased the evicted population [15, 22]. In the absence of state support, families and the voluntary sector (eg, Caritas, parishes] provided material assistance, while affected citizens joined a network of non-governmental organizations to protest and provide legal and social support to families threatened of eviction. In Spain, the main support platform is Stop Evictions-Platform of Affected by MortgagePAH [Stop Desahucios-Plataforma de Afectados por la Hipoteca-PAH] [40, 42].

The risk of suicide is often used as the probability that a person commits suicide or executes some suicidal behavior. Several factors are known to increase this risk. Among them are factors associated with the household or the subject, such as demographics, socioeconomic and employment conditions, family history, or mental health conditions, as well as stressful life events, such as serious economic and legal difficulties, as in an eviction process. On the other hand, social and family support are well-known protective factors against suicide $[43,44]$.

The present study investigated suicide risk in 205 households threatened with eviction ( 674 members) by conducting one-on-one interviews with attendees of Stop Evictions meetings in Granada, Spain. Granada, in the southern region of Andalusia, has high unemployment rates and among the highest suicide rates in Spain $[10,11,45]$. The goal of this study was to assess whether suicide risk was associated with specific features of the household (interviewee- or household-related), of the eviction process, or of support networks. Specifically, did suicide risk depend on the specific conditions of the individuals or their households? Did the duration of the eviction process and the attitude of the banks (including the two types of banks that grant mortgages in Spain, private commercial banks and public savings banks-cajas de ahorros) influence the suicide risk of mortgage victims? What was the role of social support: did formal, informal, and personal support networks mitigate suicide risk? 


\section{Methods}

\section{Context}

The data belong to a wider cross-sectional study on the impact of evictions on health. A survey assessed physical and mental health, features of the eviction process and of support networks, and use of health services. Findings on self-perceived general and mental health -PTSD, depression, and anxiety symptomatology- were reported elsewhere [22, 30, 46]. The field work was conducted between April 2013 and May 2014. Trained professionals individually administered the survey during the weekly meetings of the Stop Evictions platform that took place in Granada, Spain. Immediately before the meetings, attendants currently threatened with eviction at the time were presented the study and invited to participate, and those who agreed were interviewed in the following meeting. All participants provided written informed consent and were informed that their responses were confidential. The research project was approved by the Andalusian Health System Research Ethics Committee.

\section{Sample}

Participants were recruited among attendees of the weekly meetings of the Granada Stop Evictions platform. For the recruitment, we followed (but not strictly) the methodology called "Respondent-driven sampling", given its suitability for hard-to-reach groups [47, 48]. The recruitment began in the moments before the weekly meetings of the assemblies, and the study was presented to the attendees and they were invited to participate. From there, subjects were recruited for several weeks. The sampling process ended when the size of the sample approached the target number initially estimated, and the number of subjects recruited was so small that it indicated the end of the subjects available or willing to participate.

The interviewees were 205 adult mortgage victims (> 18 years), each representing a household at any stage of the eviction process -from mortgage arrears to having been evicted-. For instance, if three adult household members attended the meeting, only one was interviewed. Individuals who attended the meetings but were not under the threat of eviction could not take part in the study.

\section{Variables}

Our main focus was suicide risk. For this purpose the MINI International Psychiatric Interview [49] was used to identify mortgage victims showing no risk, low risk, moderate risk, or high risk of suicide. Due to the small size of the sample, the variable "risk of suicide" was dichotomized to compare victims at risk of suicide (low, moderate, high) with victims who did not show risk on that scale.
The survey, available upon request, investigated whether suicide risk was modified by the following characteristics of: 1) the household; 2) the eviction process -its duration and banks' attitudes-; 3) social support-perceived, solicited, and received, use of health care services, unmet support and health needs.

\section{Household}

Household features included 1) characteristics of the interviewee: demographics sex, age, civil status, educational level, employment, occupational social class based on the 2011 Spanish classification of occupations [50], and provider role; 2) characteristics of the household: household size, current monthly income, perceived financial strain ["each month, how hard is it to make ends meet?"), and the presence of children or disabled members.

\section{Eviction process}

To investigate whether banks contributed to mortgage victims' suicide risk, the survey evaluated 1) the stages and duration of the eviction -that is, whether the household was in arrears or in advanced stages of the eviction process and whether the household had been exposed recently or for more than 2 years to the threat of eviction. These variables allowed us to examine whether longer exposure to the eviction process was associated with a greater suicide risk. 2) Banks' attitude during phone calls -whether banks adopted a threatening rather than conciliating attitude while soliciting mortgage payback.

\section{Perceived support and support networks}

The Duke-UNK Functional Social Support Scale [51] was employed to investigate mortgage victims' perceived social support. The scale distinguishes subjectively high or low social support and identifies whether the individual feels supported or not rather than the actual support received.

Actual support was investigated by assessing the formal, informal, and personal social support networks available. In this study, formal support networks refer here to health care services, informal support networks refer to nongovernmental organizations such as parishes, Caritas, and the Stop Evictions platform, and social support networks consist of family and friends. For each type of support network, we investigated, if support was solicited, which support network -formal, informal, or personal- was the main support provider, the type of support received-material, emotional, or legal- and unmet support needs.

As for health care services, the following were analyzed: 1) Emergency Room [ER] visits in the last year, 2) primary care visits in the last 2 weeks and the type of health professional consulted (physician, psychologist or 
other), 3) unmet health care needs ["health care services you would have needed to use but could not").

\section{Statistical analysis}

All statistical analyses were performed using SPSS 25.0 [Chicago, IL, USA]. The characteristics of the sample were first examined using descriptive statistics. Next, using Pearson's $x^{2}$ or Fisher's exact test when appropriate, it was investigated whether the presence of suicide risk was associated to specific features of the interviewee/household, eviction process, and support networks.

\section{Results}

\section{Sample characteristics}

Table 1 shows the suicide risk levels and demographic, employment, and household features of the sample. The data correspond to the 205 participants in the study, representing one household each, with 673 being the total number of members of these households.

The majority of interviewees were women (59.9\%). Almost half of interviewees (46.7\%) were at low (11.8\%), moderate $(16.9 \%)$, or high suicide risk $(17.9 \%)$; and onethird thought about suicide (33.3\%).

One third of interviewees were older than 36 years. Half of the interviewees had a primary education (50.7\%), the other half had a secondary $(39.8 \%)$ or university education (9.5\%). Most men were unemployed (75\%), whereas among women $54.4 \%$ were unemployed, $18.4 \%$ were in precarious employment, $11.4 \%$ had permanent contracts, and 9.6\% were homemakers. Approximately one-third of mortgage victims belonged to the II, III, and IV occupational class and half were providers. Most households comprised more than three members. Almost half lived on less than 500 euros/month (44.15\%), the rest lived on 501 to 1000 (44.15\%) or more than 1000 euros/month (18.6\%). Virtually all households reported difficulties making ends meet (97.85\%). Most households included children (62.15\%) and almost onefifth included disabled members (18.10\%).

\section{Household and suicide risk}

Table 2 shows suicide risk as a function of the interviewee and the household features. In general, there was no specific association, however in men employment and occupational social class tended to be associated with suicide risk (both $p<0.05$ ).

\section{Eviction process and suicide risk}

As Table 3 shows, all features of the eviction process were associated with suicide risk, especially in women. Female mortgage victims, in the late stages of the eviction process, had a greater suicide risk $(p<0.05)$, whereas for men, suicide risk was similar in early and late stages of the eviction process. Similarly, the duration of the eviction process was clearly associated with suicide risk $(p<0.001)$, so that in a high percentage of men $(p<0.01)$ and women $(p<0.05)$ suicide risk increased with prolonged exposure to the eviction process. Furthermore, the attitude of banks correlated with suicide risk, so that threatening phone calls from banks were associated with a greater suicide risk $(p<0.05)$, especially in males $(p<0.05)$.

\section{Social support and suicide risk}

Based on Duke's-UNC scale, most mortgage victims $(66.3 \%)$ felt high social support, $74.7 \%$ in men, and $60.7 \%$ in women.

Table 4 shows that suicide risk was associated with low social support $(p<0.01)$, especially among women $(p<0.01)$.

Mortgage victims solicited support especially to family and friends (62.1\%), to formal support networks, i.e., social and health care (50.3\%), and to a lesser extent to informal support networks such as nongovernmental organizations $(32.3 \%)$. When at risk of suicide, interviewees solicited mostly formal support $(p<0.01)$, especially if they were men $(p>0.05)$. Consistently, men at risk of suicide solicited less help from families and friends (47.4\%) than men who were not at risk of suicide (71.4\%). As for the actual support received, family and friends were the main source of help when the evicted solicited it (86.8\%). Interviewees at risk of suicide received more help from nongovernmental organizations than those who were not at risk $(81 \%, p<0.05)$. There was no difference in the amount of formal support received between those at risk of suicide and those without risk.

Regardless of the source of support, interviewees received mostly material, i.e., money, food (66.5\%), and emotional help (50.5\%). Only emotional help was associated with suicide risk in men $(p<0.01): 70 \%$ of men at risk reported insufficient emotional help. As for unmet support needs, interviewees at risk disclosed a greater amount of unmet needs $(p<0.001)$, especially if they were women $(p<0.01)$. The main unmet need was support in mediating with banks (43.1\%), followed by psychological, emotional, and legal help. In interviewees at risk, the main unmet needs were emotional $(p<$ $0.001)$ and psychological help $(p<0.01)$, especially in men $(p<0.01$ and $p<0.05$, respectively).

Table 5 describes the use of health care services, unmet health care needs, and their interaction with mortgage victims' suicide risk.

There was a tendency for the evicted at risk of suicide to visit ER and primary care more often than those not at risk $(p<0.10)$, especially among women $(p<0.10)$. Regardless suicide risk, half of mortgage victims (53.4\%) 
Table 1 Suicide risk, Demographics, employment, and household features

\begin{tabular}{llll}
\hline Men & Women & Total \\
& $83(40.5 \%)$ & $122(59.9 \%)$ & \\
\hline
\end{tabular}

Suicide Risk (levels) ${ }^{a}$

$\begin{array}{llll}\text { No risk } & 42(52.5 \%) & 62(53.9 \%) & 104(53.3 \%) \\ \text { Low risk } & 10(12.5 \%) & 13(11.3 \%) & 23(11.8 \%) \\ \text { Moderate risk } & 17(21.3 \%) & 16(13.9 \%) & 33(16.9 \%) \\ \text { High risk } & 11(13.8 \%) & 24(10.9 \%) & 35(17.9 \%) \\ \text { Total } & 80(100 \%) & 115(100 \%) & 195(100 \%)\end{array}$

Age

$$
\begin{aligned}
& 25 \text { to } 35 \text { years } \\
& 36 \text { to } 50 \text { years } \\
& 51 \text { to } 74 \text { years } \\
& \text { Total }
\end{aligned}
$$

Civil status

Married

Unmarried

Separated/Divorced

Widowed

Total

Education level

Primary
Secondary
University
Total

Employment

Self-employed
Permanent contract
Precarious employment ${ }^{\mathrm{b}}$
Unemployed
Retired/invalidity pension
Homemaker
Total

Occupational social class ${ }^{c}$

I
II
IV
Total

Provider

$$
\begin{aligned}
& \text { Yes } \\
& \text { No }
\end{aligned}
$$$$
\text { Total }
$$

Household size

$$
<2 \text { members }
$$

3 to 5 members

$\begin{array}{lll}29(34.9 \%) & 29(23.8 \%) & 58(28.3 \%) \\ 36(43.4 \%) & 68(55.7 \%) & 104(50.7 \%) \\ 18(21.7 \%) & 25(20.5 \%) & 43(21.0 \%) \\ 83(100 \%) & 122(100 \%) & 205(100 \%)\end{array}$

43 (54.4\%)

25 (31.6\%)

11 (13.9\%)

$0(0 \%)$

79 (100\%)

$42(50.6 \%)$

37 (44.6\%)

$4(4.8 \%)$

83 (100\%)

$3(3.7 \%)$

$4(4.9 \%)$

$6(7.4 \%)$

$61(75.3 \%)$

$6(7.4 \%)$

1 (1.2\%)

81 (100\%)

$$
\begin{aligned}
& 6(7.5 \%) \\
& 33(41.3 \%) \\
& 25(31.3 \%) \\
& 16(20 \%)
\end{aligned}
$$

$59(49.2 \%)$

$102(51.3 \%)$

27 (22.5\%)

28 (23.3\%)

$6(5 \%)$

$120(100 \%)$

61 (50.8\%)

42 (35\%)

$17(14.2 \%)$

120 (100\%)

52 (26.1\%)

$39(19.6 \%)$

$6(3.0 \%)$

199 (100\%)

$103(50.7 \%)$

79 (38.9\%)

21 (10.3\%)

203 (100\%)

$4(3.5 \%)$

7 (3.6\%)

$13(11.4 \%)$

21 (18.4\%)

17 (8.7\%)

$27(13.8 \%)$

62 (54.4\%)

$123(63.1 \%)$

58 (2.6\%)

64 (32.8\%)

$12(6.2 \%)$

195 (100\%)

$114(100 \%)$

$80(100 \%)$

$47(57 \%)$

$59(50 \%)$

$36(43 \%)$

59 (50\%)

$83(100 \%)$

$118(100 \%)$

$11(5.8 \%)$

61 (31.9\%)

61 (31.9\%)

$58(30.4 \%)$

$191(100 \%)$

106 (52.7\%)

$95(47.3 \%)$

$201(100 \%)$

$23(28 \%)$

$33(27.3 \%)$

$56(27.6 \%)$

$57(69.5 \%)$
$80(66.1 \%)$
Table 1 Suicide risk, Demographics, employment, and household features (Continued)

\begin{tabular}{ccll}
\hline & $\begin{array}{l}\text { Men } \\
83(40.5 \%)\end{array}$ & $\begin{array}{l}\text { Women } \\
122(59.9 \%)\end{array}$ & Total \\
\hline$>5$ members & $2(2.4 \%)$ & $8(6.6 \%)$ & $10(4.9 \%)$ \\
Total & $82(100 \%)$ & $121(100 \%)$ & $203(100 \%)$ \\
Current monthly income (euros) & & \\
$<500$ & $48(45.8 \%)$ & $51(42.5 \%)$ & $99(48.8 \%)$ \\
$501-1000$ & $28(33.7 \%)$ & $49(40.8 \%)$ & $77(37.9 \%)$ \\
$>1000$ & $17(20.5 \%)$ & $20(16.7 \%)$ & $37(18.2 \%)$ \\
Total & $83(100 \%)$ & $120(100 \%)$ & $203(100 \%)$
\end{tabular}

Perceived financial strain (monthly)

$\begin{array}{llll}\text { Hard } & 79(97.5 \%) & 110(98.2 \%) & 189(97.9 \%) \\ \text { Easy } & 2(2.5 \%) & 2(1.8 \%) & 4(2.1 \%) \\ \text { Total } & 81(100 \% \%) & 112(100 \% \%) & 193(100 \%)\end{array}$

Children in the house

$\begin{array}{llll}\text { Yes } & 45(59.2 \%) & 71(65.1 \%) & 116(62.7 \%)\end{array}$

No $\quad 31(40.8 \%) \quad 38(34.9 \%) \quad 69(37.3 \%)$

Total $76(100 \%) \quad 109(100 \%) \quad 185(100 \%)$

Disabled members in the house

\begin{tabular}{llll} 
Yes & $12(15 \%)$ & $25(21.2 \%)$ & $37(18.7 \%)$ \\
No & $68(85 \%)$ & $93(78.8 \%)$ & $161(81.3 \%)$ \\
Total & $80(100 \%)$ & $118(100 \%)$ & $198(100 \%)$ \\
\hline
\end{tabular}

${ }^{a}$ MINI International Psychiatric Interview [47]

${ }^{\mathrm{b}}$ Precarious employment: temporary, insecure and flexible employment

c Domingo-Salvany et al., 2013 [48]

visited the ER during the past year and almost 40\% visited a health professional in the past 2 weeks. Among individuals at risk who visited a health professional, most visited a physician (69.4\%), and only $6.9 \%$ visited a psychologist. As for unmet health needs, almost twothirds of male interviewees at risk of suicide would have liked to visit a physician $(\mathrm{p}<0.05)$ or a mental health professional $(\mathrm{p}<0.05)$ but did not.

\section{Discussion}

The data suggest that suicide risk was higher among the evicted: almost $50 \%$ of mortgage victims (46.7\%) had a low (11.8\%), moderate $(16.9 \%)$, or high suicide risk (17.9\%). A European study (COURAGE) estimated the prevalence of suicide ideation, planning, and intent among adult Spaniards at 3.7, 1.9, and 1.5\% [52]. Another study (ESEMED) likewise estimated the Spanish prevalence of suicide ideas and attempts at 4.4 and $1.6 \%$ [53]. Naturally, it is problematic to compare data collected at different times using different measures, however, suicide risk appears clearly more frequently among mortgage victims. A Swedish study similarly observed that the risk of suicide was four times more frequent among the evicted [28]. 
Table 2 Interviewee and household features and suicide risk

\begin{tabular}{|c|c|c|c|c|c|c|c|c|c|c|c|c|}
\hline & \multicolumn{12}{|l|}{ Suicide risk } \\
\hline & \multicolumn{4}{|l|}{$\begin{array}{l}\text { Men } \\
\text { n (\%) }\end{array}$} & \multicolumn{4}{|l|}{$\begin{array}{l}\text { Women } \\
\mathrm{n}(\%)\end{array}$} & \multicolumn{4}{|l|}{$\begin{array}{l}\text { Total } \\
\text { n (\%) }\end{array}$} \\
\hline & No & Yes & Total & $\bar{p}$ & No & Yes & Total & $\mathrm{p}$ & No & Yes & Total & $\mathrm{p}$ \\
\hline \multicolumn{13}{|l|}{ Age } \\
\hline 25 to 35 years & 15 (35.7\%) & $12(31.6 \%)$ & $27(33.8 \%)$ & & $17(27.4 \%)$ & $11(20.8 \%)$ & $28(24.3 \%)$ & & $32(30.8 \%)$ & $23(25.3 \%)$ & $55(28.2 \%)$ & \\
\hline 36 to 50 years & $19(45.2 \%)$ & $17(44.7 \%)$ & $36(45.0 \%)$ & & $34(54.8 \%)$ & $29(54.7 \%)$ & $63(54.8 \%)$ & & $53(51.0 \%)$ & $46(50.5 \%)$ & 99 (50.8\%) & \\
\hline 51 to 74 years & $8(19.0 \%)$ & $9(23.7 \%)$ & $17(21.3 \%)$ & & $11(17.7 \%)$ & $13(24.5 \%)$ & $24(20.9 \%)$ & & $19(18.3 \%)$ & $22(24.2 \%)$ & $41(21.0 \%)$ & \\
\hline \multicolumn{13}{|l|}{ Civil status } \\
\hline Married & $21(52.5 \%)$ & $20(55.6 \%)$ & 41 (53.9\%) & & $32(52.5 \%)$ & $24(45.3 \%)$ & $56(49.1 \%)$ & & $53(52.5 \%)$ & $44(49.4 \%)$ & $97(51.1 \%)$ & \\
\hline Unmarried & $11(27.5 \%)$ & $13(36.1 \%)$ & $24(31.6 \%)$ & & $14(23.0 \%)$ & $13(24.5 \%)$ & $27(23.7 \%)$ & & $25(24.8 \%)$ & $26(29.2 \%)$ & $51(26.8 \%)$ & \\
\hline Separated; divorced; widowed. & $8(20.0 \%)$ & $3(8.3 \%)$ & $11(14.5 \%)$ & & $15(24.6 \%)$ & $16(30.2 \%)$ & $31(27.2 \%)$ & & $23(22.8 \%)$ & $19(21.3 \%)$ & $42(22.1 \%)$ & \\
\hline \multicolumn{13}{|l|}{ Education level } \\
\hline Primary & $23(54.8 \%)$ & $28(73.7 \%)$ & $51(63.8 \%)$ & & 39 (62.9\%) & $29(55.8 \%)$ & $68(59.6 \%)$ & & $62(59.6 \%)$ & $57(63.3 \%)$ & 119 (61.3\%) & \\
\hline Secondary & $16(38.1 \%)$ & $9(23.7 \%)$ & $25(31.3 \%)$ & & $14(22.6 \%)$ & $18(34.6 \%)$ & $32(28.1 \%)$ & & $30(28.8 \%)$ & $27(30.0 \%)$ & $57(29.4 \%)$ & \\
\hline University & $3(7.1 \%)$ & $1(2.6 \%)$ & $4(5.0 \%)$ & & $9(14.5 \%)$ & $5(9.6 \%)$ & $14(12.3 \%)$ & & $12(11.5 \%)$ & $6(6.7 \%)$ & $18(9.3 \%)$ & \\
\hline \multicolumn{13}{|l|}{ Employment } \\
\hline Self-employed & $2(4.8 \%)$ & $1(2.8 \%)$ & $3(3.8 \%)$ & & $1(1.7 \%)$ & $3(6.1 \%)$ & $4(3.7 \%)$ & & $3(3.0 \%)$ & $4(4.7 \%)$ & $7(3.8 \%)$ & \\
\hline Permanent contract & $3(7.1 \%)$ & $1(2.8 \%)$ & $4(5.1 \%)$ & & $6(10.2 \%)$ & $5(10.2 \%)$ & $11(10.2 \%)$ & & $9(8.9 \%)$ & $6(7.1 \%)$ & $15(8.1 \%)$ & \\
\hline Temporary contract & $0(0.0 \%)$ & $6(16.7 \%)$ & $6(7.7 \%)$ & & $9(15.3 \%)$ & $12(24.5 \%)$ & $21(19.4 \%)$ & & $9(8.9 \%)$ & $18(21.2 \%)$ & $27(14.5 \%)$ & \\
\hline Unemployed & $33(78.6 \%)$ & $26(72.2 \%)$ & $59(75.6 \%)$ & & $35(59.3 \%)$ & $24(49.0 \%)$ & $59(54.6 \%)$ & & $68(67.3 \%)$ & $50(58.8 \%)$ & 118 (63.4\%) & \\
\hline Retired/invalidity pension & $4(9.5 \%)$ & $1(2.8 \%)$ & $5(6.4 \%)$ & & $3(5.1 \%)$ & $0(0.0 \%)$ & $3(2.8 \%)$ & & $7(6.9 \%)$ & $1(1.2 \%)$ & $8(4.3 \%)$ & \\
\hline Homemaker & $0(0.0 \%)$ & $1(2.8 \%)$ & $1(1.3 \%)$ & * & $5(8.5 \%)$ & $5(10.2 \%)$ & $10(9.3 \%)$ & & $5(5.0 \%)$ & $6(7.1 \%)$ & $11(5.9 \%)$ & t \\
\hline \multicolumn{13}{|l|}{ Occupational social class } \\
\hline 1 & $3(7.7 \%)$ & $3(7.9 \%)$ & $6(7.8 \%)$ & & $2(3.4 \%)$ & $2(4.2 \%)$ & $4(3.8 \%)$ & & $5(5.2 \%)$ & $5(5.8 \%)$ & $10(5.5 \%)$ & \\
\hline$\|$ & $17(43.6 \%)$ & $14(36.8 \%)$ & $31(40.3 \%)$ & & $17(29.3 \%)$ & $9(18.8 \%)$ & $26(24.5 \%)$ & & $34(35.1 \%)$ & $23(26.7 \%)$ & $57(31.1 \%)$ & \\
\hline III & $7(17.9 \%)$ & $17(44.7 \%)$ & $24(31.2 \%)$ & & $16(27.6 \%)$ & $19(39.6 \%)$ & 35 (33.0\%) & & $23(23.7 \%)$ & $36(41.9 \%)$ & $59(32.2 \%$ & \\
\hline IV & $12(30.8 \%)$ & $4(10.5 \%)$ & $16(20.8 \%)$ & * & $23(39.7 \%)$ & $18(37.5 \%)$ & $41(38.7 \%)$ & & $35(36.1 \%)$ & $22(25.6 \%)$ & $57(31.1 \%)$ & t \\
\hline \multicolumn{13}{|l|}{ Provider } \\
\hline Yes & $22(52.4 \%)$ & $24(63.2 \%)$ & $46(57.5 \%)$ & & $30(48.4 \%)$ & $25(50.0 \%)$ & $55(49.1 \%)$ & & $52(50.0 \%)$ & $49(55.7 \%)$ & $101(52.6 \%)$ & \\
\hline No & $20(47.6 \%)$ & $14(36.8 \%)$ & $34(42.5 \%)$ & & $32(51.6 \%)$ & $25(50.0 \%)$ & $57(50.9 \%)$ & & $52(50.0 \%)$ & 39 (44.3\%) & $91(47.4 \%)$ & \\
\hline \multicolumn{13}{|l|}{ Household size } \\
\hline$<2$ members & $14(33.3 \%)$ & $9(24.3 \%)$ & $23(29.1 \%)$ & & $13(21.0 \%)$ & $19(35.8 \%)$ & $32(27.8 \%)$ & & $27(26.0 \%)$ & $28(31.1 \%)$ & $55(28.4 \%)$ & \\
\hline 3 to 5 members & $28(66.7 \%)$ & $26(70.3 \%)$ & $54(68.4 \%)$ & & $45(72.6 \%)$ & $30(56.6 \%)$ & $75(65.2 \%)$ & & $73(70.2 \%)$ & $56(62.2 \%)$ & $129(66.5 \%)$ & \\
\hline$>5$ members & $0(0.0 \%)$ & $2(5.4 \%)$ & $2(2.5 \%)$ & & $4(6.5 \%)$ & $4(7.5 \%)$ & $8(7.0 \%)$ & & $4(3.8 \%)$ & $6(6.7 \%)$ & $10(5.2 \%$ & \\
\hline \multicolumn{13}{|l|}{ Current monthly income (euros) } \\
\hline$<500$ & $21(50.0 \%)$ & $16(42.1 \%)$ & $37(46.3 \%)$ & & $26(41.9 \%)$ & $22(43.1 \%)$ & $48(42.5 \%)$ & & $47(45.2 \%)$ & $38(42.7 \%)$ & $85(44.0 \%)$ & \\
\hline $501-1000$ & $12(28.6 \%)$ & $14(36.8 \%)$ & $26(32.5 \%)$ & & $26(41.9 \%)$ & $23(45.1 \%)$ & $49(43.4 \%)$ & & $38(36.5 \%)$ & $37(41.6 \%)$ & 75 (38.9\%) & \\
\hline$>1000$ & $9(21.4 \%)$ & $8(21.1 \%)$ & $17(21.3 \%)$ & & $10(16.1 \%)$ & $6(11.8 \%)$ & $16(14.2 \%)$ & & $19(18.3 \%)$ & $14(15.7 \%)$ & $33(17.1 \%)$ & \\
\hline \multicolumn{13}{|l|}{ Perceived financial strain (monthly) } \\
\hline Hard & $39(97.5 \%)$ & $37(97.4 \%)$ & $71(97.3 \%)$ & & $57(100.0 \%)$ & $48(98.0 \%)$ & $105(99.1 \%)$ & & $96(99.0 \%)$ & $85(97.7 \%)$ & $181(98.4 \%)$ & \\
\hline Easy & $1(2.5 \%)$ & $1(2.6 \%)$ & $2(2.7 \%)$ & & $0(0 \%)$ & $1(2.0 \%)$ & $1(0.9 \%)$ & & $1(1.0 \%)$ & $2(2.3 \%)$ & $3(1.6 \%)$ & \\
\hline \multicolumn{13}{|l|}{ Children in the house } \\
\hline Yes & $23(59.0 \%)$ & $21(60.0 \%)$ & $44(59.5 \%)$ & & $41(70.7 \%)$ & $26(56.5 \%)$ & $67(64.4 \%)$ & & $64(66.0 \%)$ & $47(58.0 \%)$ & $111(62.4 \%)$ & \\
\hline No & $16(41.0 \%)$ & $14(40.0 \%)$ & $30(40.5 \%)$ & & $17(29.3 \%)$ & $20(43.5 \%)$ & $37(35.6 \%)$ & & $33(34.0 \%)$ & $34(42.0 \%)$ & $67(37.6 \%)$ & \\
\hline \multicolumn{13}{|l|}{ Disabled members in the house } \\
\hline Yes & $6(14.3 \%)$ & $6(17.1 \%)$ & $12(15.6 \%)$ & & $10(16.7 \%)$ & $14(26.9 .3 \%)$ & $24(21.4 \%)$ & & $16(15.7 \%)$ & $20(23.0 \%)$ & $36(40.4 \%)$ & \\
\hline No & 36 (85.7\%) & $29(82.9 \%)$ & 65 (84.4\%) & & $50(83.3 \%)$ & 38 (73.1\%) & $88(78.6 \%)$ & & 86 (84.3\%) & 67 (77.0\%) & $53(59.6 \%)$ & \\
\hline
\end{tabular}

$\dagger p<.10{ }^{*} p<.05{ }^{* *} p<.01{ }^{* * *} p<.001$ 
Table 3 Eviction process, banks' attitude, and suicide risk

\begin{tabular}{|c|c|c|c|c|c|c|c|c|c|c|c|c|}
\hline & \multicolumn{12}{|l|}{$\underline{\text { Suicide risk }}$} \\
\hline & \multicolumn{4}{|l|}{$\begin{array}{l}\text { Men } \\
\mathrm{n}(\%)\end{array}$} & \multicolumn{4}{|l|}{$\begin{array}{l}\text { Women } \\
\mathrm{n}(\%)\end{array}$} & \multicolumn{4}{|l|}{$\begin{array}{l}\text { Total } \\
\text { n (\%) }\end{array}$} \\
\hline & No & Yes & Total & $\mathrm{p}$ & No & Yes & Total & $\mathrm{p}$ & No & Yes & Total & $\mathrm{p}$ \\
\hline \multicolumn{13}{|l|}{ Stage in eviction } \\
\hline In arrears & $20(50.0 \%)$ & $19(51.4 \%)$ & $39(50.6 \%)$ & & $43(72.9 \%)$ & $23(50.0 \%)$ & $65(62.5 \%)$ & & $63(63.6 \%)$ & $42(50.6 \%)$ & $105(57.7 \%)$ & \\
\hline Ongoing eviction & $20(50.0 \%)$ & $18(48.6 \%)$ & $38(49.4 \%)$ & & $16(27.1 \%)$ & $23(50.0 \%)$ & $39(37.5 \%)$ & * & $36(36.4 \%)$ & $41(49.4 \%)$ & $77(42.3 \%)$ & t \\
\hline \multicolumn{13}{|c|}{ Duration of the eviction process } \\
\hline Recent & $32(88.9 \%)$ & $22(62.9 \%)$ & $54(76.1 \%)$ & & 37 (74.0\%) & $26(53.1 \%)$ & $63(63.6 \%)$ & & $69(80.2 \%)$ & $48(57.1 \%)$ & $117(68.8 \%)$ & \\
\hline$>2$ years & $4(11.1 \%)$ & $13(37.1 \%)$ & $17(23.9 \%)$ & ** & $13(26.0 \%)$ & $23(46.9 \%)$ & $36(36.4 \%)$ & * & 17 (19.8\%) & $36(42.9 \%)$ & $53(31.2 \%)$ & $* * *$ \\
\hline \multicolumn{13}{|l|}{$\begin{array}{l}\text { Banks' attitude during } \\
\text { phone calls }\end{array}$} \\
\hline Conciliating & $15(68.2 \%)$ & $8(36.4 \%)$ & $23(52.3 \%)$ & & $18(64.3 \%)$ & $18(51.4 \%)$ & $36(57.1 \%)$ & & $33(66.0 \%)$ & $26(45.6 \%)$ & $59(50.9 \%)$ & \\
\hline Threatening & 7 (31.8\%) & 14 (63.6\%) & $21(47.7 \%)$ & * & 10 (35.7\%) & 17 (48.6\%) & $27(42.4 \%)$ & * & 17 (34.0\%) & 31 (54.4\%) & $57(49.1 \%)$ & * \\
\hline
\end{tabular}

Specifically, the present study investigated whether the risk of suicide among mortgage victims was associated with specific characteristics of the household, the interviewee, the eviction process and the support networks. The main findings were the following: household/interviewee features were scarcely associated to suicide risk, and only among men, worse employment conditions and lower social class increased suicide risk. This finding can be interpreted in the context of Spain gender inequalities and, specifically, the social expectations of men being the breadwinners [54]. When banks adopted a threatening attitude, the risk of suicide increased among mortgage victims. To our knowledge, this is the first study documenting the role of banks in mortgage victims' suicide risk. As for social support, it was inversely related to suicide risk, being less frequent when the evicted felt socially supported. Findings on solicited, received, and unmet support and health needs pinpoint the specific lacks and needs of the evicted. Mortgage victims at risk of suicide solicited help especially from family/friends and formal networks but received it more frequently from family and nongovernmental organizations. For mortgage victims as a whole, family and friends constituted the main source of help actually received, in line with other studies showing that social networks provided around $80 \%$ of long-term care [55]. The main unmet support needed among mortgage victims was the negotiation with banks. However, people in the process of eviction who were at risk of suicide showed greater unmet needs for emotional and psychological help, than those who were not at risk of suicide. The use of health care services was higher among mortgage victims compared to the general population [56], confirming that mortgage victims searched for help in the formal healthcare system. Nonetheless, in case of suicide risk, more than two-thirds of the evicted visited a physician and identified visits to physicians or mental health professionals as the main unmet health needs.

\section{The role of social support}

In recent decades, psychophysiological research has found a consistent relationship between social support -and its reverse, loneliness- and health: strong social support benefits the cardiovascular, endocrine, and immune systems, whereas loneliness and social isolation are associated with "greater mortality than wellestablished risk factors, such as cigarette smoking" [57]. Social isolation is accompanied by greater suicide risk, in adults [58-61], as well as in older men exposed to the recent economic crisis [62]. Moreover, suicide risk increases when individuals feel lonely [50, 60, 61]. Emotional support has been identified as a key dimension of social support [57], and in the current study it emerged as a key risk factor of suicide: suicide risk was less frequent when mortgage victims felt that they received sufficient emotional help, and mortgage victims at suicide risk identified emotional and psychological help as the main unmet needs.

\section{The role of primary care}

Several professional associations have advocated the need for a formal network of support to the evicted [63-65]. In addition, attention has been repeatedly drawn to the possible very negative effects on the health of victims of evictions, suggesting the organization of community health programs at the level of primary care and considering that this level of health care is capable of managing and organizing effective interventions on mental health [66-68].

Previous publications from the World Health Organization [WHO] confirm this, further adding that "shifting mental health care to primary health care 
Table 4 Social support and suicide risk. Support perceived, solicited, received, and unmet support needs

\begin{tabular}{|c|c|c|c|c|c|c|c|c|c|c|c|c|}
\hline & \multicolumn{12}{|l|}{ Suicide risk } \\
\hline & \multicolumn{4}{|l|}{$\begin{array}{l}\text { Men } \\
\text { n (\%) }\end{array}$} & \multicolumn{4}{|l|}{$\begin{array}{l}\text { Women } \\
\mathrm{n}(\%)\end{array}$} & \multicolumn{4}{|l|}{$\begin{array}{l}\text { Total } \\
\text { n (\%) }\end{array}$} \\
\hline & No & Yes & Total & $\mathrm{p}$ & No & Yes & Total & $\mathrm{p}$ & No & Yes & Total & $p$ \\
\hline \multicolumn{13}{|c|}{ Support perceived (Duke's-UNC scale) } \\
\hline High & $33(78.6 \%)$ & $27(71.1 \%)$ & $60(75 \%)$ & & $46(74.2 \%)$ & $23(43.4 \%)$ & $69(60 \%)$ & & 79 (76\%) & $50(54.9 \%)$ & $129(66.2 \%)$ & \\
\hline Low & $9(21.4 \%)$ & $11(28.9 \%)$ & $20(25.0 \%)$ & & $16(25.8 \%)$ & $30(56.6 \%)$ & $46(40 \%)$ & $* * *$ & $25(24 \%)$ & $41(45.1 \%)$ & 66 (33.8\%) & ** \\
\hline \multicolumn{13}{|c|}{ Source of support solicited ${ }^{a}$} \\
\hline \multicolumn{13}{|c|}{ Formal support networks } \\
\hline No & $28(66.7 \%)$ & $16(42.1 \%)$ & $44(55 \%)$ & & $33(53.2 \%)$ & $20(37.7 \%)$ & $53(46.1 \%)$ & & $61(58.7 \%)$ & $36(39.6 \%)$ & $97(49.7 \%)$ & \\
\hline Yes & $14(33.3 \%)$ & $22(57.9 \%)$ & $36(45.0 \%)$ & * & $29(46.8 \%)$ & $33(63 . \%)$ & $62(53.9 \%)$ & + & $43(41.3 \%)$ & $55(60.4 \%)$ & $98(50.3 \%)$ & $* *$ \\
\hline \multicolumn{13}{|c|}{ Informal support networks } \\
\hline No & $32(76.2 \%)$ & $26(68.4 \%)$ & $58(72.5 \%)$ & & $42(67.7 \%)$ & $32(60.4 \%)$ & $74(64.3 \%)$ & & $74(71.4 \%)$ & $58(63.7 \%)$ & $132(67.7 \%)$ & \\
\hline Yes & $10(23.8 \%)$ & $12(31.6 \%)$ & $22(27.5 \%)$ & & $20(32,3 \%)$ & $21(39.6 \%)$ & $41(35.7 \%)$ & & $30(28.8 \%)$ & $33(36.3 \%)$ & $63(32.3 \%)$ & \\
\hline \multicolumn{13}{|l|}{ Family/friends } \\
\hline No & $12(28.6 \%)$ & $20(52.6 \%)$ & $32(40.0 \%)$ & & $25(40.3 \%)$ & 17 (32.1\%) & $42(36.5 \%)$ & & $37(35.6 \%)$ & $37(40.7 \%)$ & $74(37.9 \%)$ & \\
\hline Yes & $30(71.4 \%)$ & $18(47.4 \%)$ & $48(60.0 \%)$ & * & $37(59.7 \%)$ & $36(67.9 \%)$ & $73(63.5 \%)$ & & $67(64.4 \%)$ & $54(59.3 \%)$ & $121(62.1 \%)$ & \\
\hline \multicolumn{13}{|c|}{ Source of support received ${ }^{b}$} \\
\hline \multicolumn{13}{|c|}{ Formal support networks } \\
\hline No & $3(21.4 \%)$ & $7(31.8 \%)$ & $10(27.8 \%)$ & & $14(48.3 \%)$ & $11(33.3 \%)$ & $25(40.3 \%)$ & & $17(39.5 \%)$ & $18(32.7 \%)$ & $35(35.7 \%)$ & \\
\hline Yes & $11(78.6 \%)$ & $15(68.2 \%)$ & $26(72.2 \%)$ & & $15(51.7 \%)$ & $22(66.7 \%)$ & $37(59.7 \%)$ & & $26(60.5 \%)$ & $37(67.3 \%)$ & $63(64.3 \%)$ & \\
\hline \multicolumn{13}{|c|}{ Informal support networks } \\
\hline No & $0(0.0 \%)$ & $1(8.3 \%)$ & $1(4.5 \%)$ & & $9(45.0 \%)$ & $2(9.5 \%)$ & $11(26.8 \%)$ & & $9(30.0 \%)$ & $3(9.1 \%)$ & $12(19.0 \%)$ & \\
\hline Yes & $10(100 \%)$ & $11(91.7 \%)$ & $21(95.5 \%)$ & & $11(55,0 \%)$ & 19 (90.5\%) & $30(73.2 \%)$ & $* *$ & $21(70 \%)$ & $30(90.9 \%)$ & $51(81.0 \%)$ & * \\
\hline \multicolumn{13}{|l|}{ Family/friends } \\
\hline No & $1(3.3 \%)$ & $2(11.1 \%)$ & $3(6.3 \%)$ & & $5(13.5 \%)$ & $8(22.2 \%)$ & $13(17.8 \%)$ & & $6(9.0 \%)$ & $10(18.5 \%)$ & $16(13.2 \%)$ & \\
\hline Yes & $29(96.7 \%)$ & $16(88.9 \%)$ & $45(93.8 \%)$ & & $32(86.5 \%)$ & $28(77.8 \%)$ & $60(82.2 \%)$ & & $61(91.0 \%)$ & $44(81.5 \%)$ & $105(86.8 \%)$ & \\
\hline \multicolumn{13}{|c|}{ Type of help received, regardless the support network } \\
\hline \multicolumn{13}{|l|}{ Material help } \\
\hline No & $14(34.0 \%)$ & $15(37.5 \%)$ & $29(35.4 \%)$ & & $25(37.9 \%)$ & $14(25.5 \%)$ & $39(32.2 \%)$ & & $39(36.1 \%)$ & $29(30.5 \%)$ & $68(33.5 \%)$ & \\
\hline Yes & $28(66.7 \%)$ & $25(62.5 \%)$ & $53(64.6 \%)$ & & $41(62.1 \%)$ & $41(74.5 \%)$ & $82(67.8 \%)$ & & $69(63.9 \%)$ & $66(69.5 \%)$ & $135(66.5 \%)$ & \\
\hline \multicolumn{13}{|l|}{ Emotional help } \\
\hline No & $16(38.1 \%)$ & $28(70.0 \%)$ & $44(53.7 \%)$ & & $32(48.5 \%)$ & $24(44.4 \%)$ & $56(46.7 \%)$ & & $48(44.4 \%)$ & $52(55.3 \%)$ & $100(49.5 \%)$ & \\
\hline Yes & $26(61.9 \%)$ & $12(30.0 \%)$ & $38(46.3 \%)$ & $* *$ & $34(51.5 \%)$ & $30(55.6 \%)$ & $64(53.3 \%)$ & & $60(55.6 \%)$ & $42(44.7 \%)$ & $102(50.5 \%)$ & \\
\hline \multicolumn{13}{|l|}{ Legal help } \\
\hline No & $33(79.6 \%)$ & $33(82.5 \%)$ & $66(80.5 \%)$ & & $48(72.7 \%)$ & $47(87.0 \%)$ & $95(79.2 \%)$ & & $81(75.0 \%)$ & $80(85.1 \%)$ & $161(79.7 \%)$ & \\
\hline Yes & $9(21.4 \%)$ & $7(17.5 \%)$ & $16(19.5 \%)$ & & $18(27.3 \%)$ & $7(13.0 \%)$ & $25(20.8 \%)$ & & $27(25.0 \%)$ & $14(14.9 \%)$ & $41(20.3 \%)$ & \\
\hline \multicolumn{13}{|c|}{ Amount of unmet support needs (up to 3 ) } \\
\hline None & $1(2.4 \%)$ & $0(0 \%)$ & $1(1.3 \%)$ & & $8(12.9 \%)$ & $4(7.5 \%)$ & $12(10.4 \%)$ & & $9(8.7 \%)$ & $4(4.4 \%)$ & $13(6.7 \%)$ & \\
\hline 1 & 19 (45.2\%) & $14(36.8 \%)$ & $33(41.3 \%)$ & & $26(41.9 \%)$ & $14(26.4 \%)$ & $40(34.8 \%)$ & & $45(43.3 \%)$ & $28(30.8 \%)$ & $73(37.4 \%)$ & \\
\hline 2 & $14(33.3 \%)$ & $10(26.3 \%)$ & $24(30.0 \%)$ & & $22(35.5 \%)$ & $14(26.4 \%)$ & $36(31.3 \%)$ & & $36(34.6 \%)$ & $24(26.4 \%)$ & $60(30.8 \%)$ & \\
\hline 3 & $8(19.0 \%)$ & $14(36.8 \%)$ & $22(27.5 \%)$ & & $6(9.7 \%)$ & $21(39.6 \%)$ & $27(23.5 \%)$ & $* *$ & $14(13.5 \%)$ & $35(38.5 \%)$ & $49(25.1 \%)$ & $* * *$ \\
\hline \multicolumn{13}{|c|}{ Type of unmet support needs. } \\
\hline \multicolumn{13}{|c|}{ Seeking an alternative house } \\
\hline Not selected & $37(88.1 \%)$ & $33(86.8 \%)$ & $70(87.5 \%)$ & & 59 (95.2\%) & $47(88.7 \%)$ & $106(92.2 \%)$ & & $96(92.3 \%)$ & $80(87.9 \%)$ & $176(90.3 \%)$ & \\
\hline Selected & $5(11.9 \%)$ & $5(13.2 \%)$ & $10(12.5 \%)$ & & $3(4.8 \%)$ & $6(11.3 \%)$ & $9(7.8 \%)$ & & $8(7.7 \%)$ & 11 (12.1\%) & 19 (9.7\%) & \\
\hline
\end{tabular}


Table 4 Social support and suicide risk. Support perceived, solicited, received, and unmet support needs (Continued)

\begin{tabular}{|c|c|c|c|c|c|c|c|c|c|c|c|c|}
\hline & \multicolumn{12}{|l|}{ Suicide risk } \\
\hline & \multicolumn{4}{|l|}{$\begin{array}{l}\text { Men } \\
n(\%)\end{array}$} & \multicolumn{4}{|l|}{$\begin{array}{l}\text { Women } \\
\mathrm{n}(\%)\end{array}$} & \multicolumn{4}{|l|}{$\begin{array}{l}\text { Total } \\
\text { n (\%) }\end{array}$} \\
\hline & No & Yes & Total & $p$ & No & Yes & Total & $\mathrm{p}$ & No & Yes & Total & $\mathrm{p}$ \\
\hline \multicolumn{13}{|c|}{ Psychological help } \\
\hline Not selected & $32(57.9 \%)$ & $24(42.1 \%)$ & $54(100 \%)$ & & $45(72.5 \%)$ & $28(52.8 \%)$ & $73(63.5 \%)$ & & 77 (74.0\%) & $50(54.9 \%)$ & $127(65.1 \%)$ & \\
\hline Selected & $10(23.8 \%)$ & $16(64.0 \%)$ & $26(32,5 \%)$ & $\dagger$ & $17(27.4 \%)$ & $25(47.2 \%)$ & $42(36.5 \%)$ & * & $27(26.0 \%)$ & $41(59.4 \%)$ & $68(34.6 \%)$ & ** \\
\hline \multicolumn{13}{|l|}{ Emotional help } \\
\hline Not selected & $34(81.0 \%)$ & $24(63.2 \%)$ & $58(72.5 \%)$ & & 49 (79.0\%) & $29(54.7 \%)$ & $78(67.8 \%)$ & & $83(79.8 \%)$ & $53(58.2 \%)$ & $136(69.7 \%)$ & \\
\hline Selected & $8(19.0 \%)$ & $14(36.8 \%)$ & $22(27.5 \%)$ & t & $13(21.0 \%)$ & $24(45.3 \%)$ & 37 (32.2\%) & $* *$ & $21(20.2 \%)$ & $38(41.8 \%)$ & $59(30.3 \%)$ & $* * *$ \\
\hline \multicolumn{13}{|c|}{ Better health and social care support } \\
\hline No & $36(85.7 \%)$ & 32 (84.2\%) & 68 (85.0\%) & & 57 (91.9\%) & $46(86.8 \%)$ & $103(89.6 \%)$ & & 80 (59.7\%) & $54(40.3 \%)$ & $134(84.8 \%)$ & \\
\hline Yes & $6(14.3 \%)$ & $6(15.8 \%)$ & $12(15.0 \%)$ & & $5(8.1 \%)$ & $7(13.2 \%)$ & $12(10.4 \%)$ & & $11(10.6 \%)$ & $13(14.3 \%)$ & $24(12.3 \%)$ & \\
\hline \multicolumn{13}{|l|}{ Legal help } \\
\hline No & $26(61.9 \%)$ & $26(68.1 \%)$ & $52(65.0 \%)$ & & $47(75.8 \%)$ & 40 (75.5\%) & 87 (75.7\%) & & 73 (70.2\%) & 66 (72.5\%) & $139(71.3 \%)$ & \\
\hline Yes & $16(38.1 \%)$ & $12(31.6 \%)$ & $28(35.0 \%)$ & & 15 (24.2\%) & $13(24.5 \%)$ & $28(24.3 \%)$ & & 31 (29.8\%) & 25 (27.5\%) & $56(28.7 \%)$ & \\
\hline \multicolumn{13}{|c|}{ Negotiation with banks } \\
\hline No & $22(52.4 \%)$ & $21(55.3 \%)$ & $43(53.8 \%)$ & & $38(61.3 \%)$ & 30 (56.6\%) & 68 (59.1\%) & & $60(57.7 \%)$ & $51(56.0 \%)$ & $111(56.9 \%)$ & \\
\hline Yes & $20(47.6 \%)$ & 17 (44.7\%) & 37 (46.3\%) & & $24(38.7 \%)$ & 23 (43.4\%) & 47 (40.9\%) & & 44 (42.3\%) & 40 (44.0\%) & 84 (43.1\%) & \\
\hline
\end{tabular}

tp $<.10 * \mathrm{p}<.05{ }^{* *} \mathrm{p}<.01{ }^{* * *} \mathrm{p}<.001$

a'To whom did you solicit support?'

b'Among solicited support networks, from whom did you receive support?'

Table $\mathbf{5}$ Use of health care services and unmet health needs

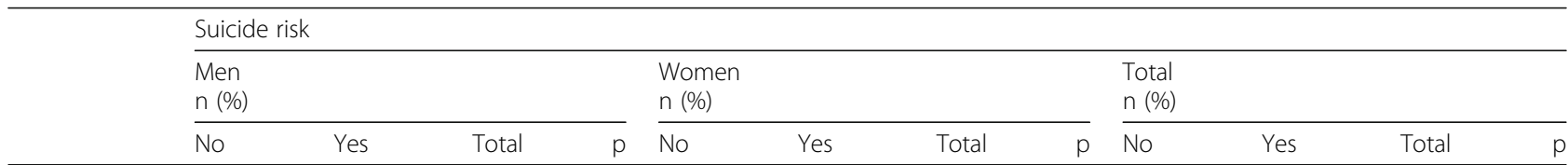

Use of health care services

ER visits (last year)

$\begin{array}{lccccccccccc}\text { No } & 24(57.1 \%) & 19(50.0 \%) & 43(53.8 \%) & 30(49.2 \%) & 17(32.7 \%) & 47(41.6 \%) & 54(52.4 \%) & 36(40.0 \%) & 90(46.6 \%) \\ \text { Yes } & 18(42.9 \%) & 19(50.0 \%) & 37(46.3 \%) & 31(50.8 \%) & 35(67.3 \%) & 66(58.4 \%) & \dagger & 49(47.6 \%) & 54(60.0 \%) & 103(53.4 \%) & \dagger \\ \text { Primary care visits (last 2 weeks) } & & & & & & & & & \\ \text { No } & 31(73.8 \%) & 24(66.7 \%) & 55(70.5 \%) & 39(63.9 \%) & 25(48.1 \%) & 64(56.6 \%) & 70(68.0 \%) & 49(55.7 \%) & 119(62.3 \%) \\ \text { Yes } & 11(26.2 \%) & 12(33.3 \%) & 23(29.5 \%) & 22(36.1 \%) & 27(51.9 \%) & 49(43.4 \%) & \dagger & 33(32.0 \%) & 39(44.3 \%) & 72(37.7 \%) & +\end{array}$

Type of health professional consulted (last 2 weeks)

$\begin{array}{llllllllll}\text { Physician } & 9(81.8 \%) & 8(66.7 \%) & 17(73.9 \%) & 14(63.6 \%) & 19(70.4 \%) & 33(67.3 \%) & 23(69.7 \%) & 27(69.2 \%) & 50(69.4 \%) \\ \text { Psychologist } & 1(9.1 \%) & 3(25 \%) & 4(17.4 \%) & 0(0.0 \%) & 1(3.7 \%) & 1(2.0 \%) & 1(3.0 \%) & 4(10.3 \%) & 5(6.9 \%) \\ \text { Other } & 1(9.1 \%) & 1(8.3 \%) & 2(8.7 \%) & 8(36.4 \%) & 7(25.9) & 15(30.6 \%) & 9(27.3 \%) & 8(20.5 \%) & 17(23.6 \%)\end{array}$

Unmet health care needs ${ }^{a}$

\begin{tabular}{|c|c|c|c|c|c|c|c|c|c|c|c|}
\hline \multicolumn{12}{|c|}{ Physician } \\
\hline No & $26(61.9 \%)$ & $13(35.1 \%)$ & $39(49.4 \%)$ & & $18(29.0 \%)$ & $13(25.0 \%)$ & $31(27.2 \%)$ & $44(42.3 \%)$ & $26(29.2 \%)$ & 70 (36.3\%) & \\
\hline Yes & $16(38.1 \%)$ & $24(64.9 \%)$ & $40(50.6 \%)$ & $*$ & $44(71.0 \%)$ & $39(75.0 \%)$ & $83(72.8 \%)$ & $60(57.7 \%)$ & $63(70.2 \%)$ & $123(63.7 \%)$ & t \\
\hline \multicolumn{12}{|c|}{ Mental health } \\
\hline No & $23(54.8 \%)$ & 11 (28.9\%) & $34(42.5 \%)$ & & $20(32.3 \%)$ & $13(24.5 \%)$ & 33 (28.7\%) & $43(41.3 \%)$ & $24(26.4 \%)$ & 67 (34.4\%) & \\
\hline Yes & 19 (45.2\%) & 27 (71.1\%) & $46(57.5 \%)$ & * & $42(67.7 \%)$ & $40(75.5 \%)$ & 82 (71.3\%) & 61 (58.7\%) & 67 (73.6\%) & 128 (65.6\%) & * \\
\hline
\end{tabular}

a'Which health care services would you have liked to use, but did not?' 
level also helps to reduce stigma, improves early detection and treatment, leads to cost efficiency and savings, and partly offsets limitations of mental health resources through the use of community resources" $[69,70]$. The current findings indicate that the evicted sought help in the health care system and visited health professionals more often than the general population, overall confirming that primary care constitutes the environment most suitable for providing formal support to the evicted. In addition, this study shows that mortgage victims need more psychological help, and that they were receptive to the help they received. Therefore, it can be assumed that support networks at the primary care level could be cost effective, while generating significant benefits for the wellbeing of mortgage victims.

\section{The role of banks}

Based on our results, banks have a clear role in mortgage victims' suicide risk: suicide risk increases with longer exposure to the eviction process and when banks adopt a threatening attitude while soliciting payback. The possible relationship between human rights violations and health was first proposed in the mid-1980s [71] and has successively been substantiated by data showing that social injustice and inequalities prompt worse health [72, 73].

The recent economic crisis and the policies adopted to stabilize and improve the economy have worsened socioeconomic inequalities. $[15,74,75]$. And inequalities have been associated with a higher suicide risk $[4,16]$.

The lack of fairness in Spanish mortgage laws has been the focus of law practitioners and academics that have advocated the need of substantial changes in mortgage laws which caused a "social drama" and constitute "a legal problem" [76, 77]. While shared ownership and temporal ownership, recently studied in Spain, have been identified as feasible alternatives in case of arrears to circumvent the rigidity of mortgage laws [78], our data indicate that the main unmet need among the evicted was help in negotiating with banks, again emphasizing the lack of formal social support that Spanish mortgage victims experienced.

\section{Dismissal of evidence on evictions and suicide}

Nonetheless, a recent 257-page-long Spanish report on "Economic crisis and health" commissioned by the Ministry of Health [79] did not mention at all, evictions or foreclosures at all, nor Spanish or international studies that describe their detrimental impact on mortgage victims' health.

In Spain, despite the call to action for the evicted from primary health care and mental health care practitioners $[64,65]$ and despite the consistency of national and international scientific literature in indicating that evictions are accompanied by depression, anxiety, and a higher suicide risk $[5,10,28,80]$, the focus still appears to be on the limited reliability of the data and on the limited evidence about the impact of the crisis on the general population [79].

Accordingly, although in the report commissioned by the Spanish Ministry of Health it was acknowledged that the crisis prompted a clear worsening in mental health and in social inequities that hit disadvantaged groups the most, evictions were not mentioned, and the impact of the crisis was substantially downplayed: "the crisis [...] does not seem to have influenced health [...], apart from mental health". The attitude toward the impact of the crisis on health has been similar in Greece: after being struck the hardest by the crisis and adopting strict austerity measures [3], findings on the adverse health consequences of the crisis were regarded as scarcely reliable and controversial [see, e.g., 82]. Such tendency to dismiss the available evidence has been described as denialism [81] and has the crucial consequence of changing priorities in state policies $[38,82]$. Thus, the financial system is favored over citizens' wellbeing, although there is reliable evidence that the worsening of mental health and the increase in suicides can be prevented or at least limited [35, 83].

\section{Methodological limitations and strengths}

The limitations of this study are due to the cross-sectional design, the limited sample size, and the focus on mortgage victims belonging to the Stop Evictions platform. However, this platform is the only movement in Granada and most cities of Spain that offers mutual and legal support to people affected by a foreclosure process. For participant recruitment, respondent-driven sampling was used, albeit not strictly, for its suitability for hardto-reach groups [48]. Moreover, sample characteristics were consistent with those observed in other Spanish studies on foreclosures $[84,85]$.

Over the 13 months of this research field work, there were about 250 foreclosures active in the courts of Granada, suggesting that the number of households included in this study (205) was adequate for the total amount of ongoing evictions [86].

In previous studies, data on suicides and evictions were mostly collected from forensic medicine registers, statistical institutions, and national databases, which render it methodologically challenging to identify and access single cases $[87,88]$, also due to the social stigma associated to both $[19,89]$. However, the current study stems from our interest in investigating, beyond the association between evictions and suicide, the factors involved in the eviction process that might mitigate or increase mortgage victims' suicide risk. 
In a study with a similar goal [28], 22,000 Swedish households were interviewed and reported that suicide risk increased by four as a result of evictions "independently of well-known suicidogenic risk factors preceding eviction". The data could be collected because the Swedish government adopted a policy of full disclosure. In Spain, the Bank of Spain -not the government- made data on home evictions available only 5 years after the crisis [78]. The link between evictions and suicides could not be investigated like in Sweden, by directly assessing all evicted households, so it was investigated by indirectly studying suicide trends in the general population [e.g., 10] or by directly accessing reduced samples like the current [22], where participants had overcome social stigma $[87,88]$ and to engage with mortgage victims groups. It can be speculated that at least part of the evicted who did not reach out for mortgage victims groups might have constituted worse cases, characterized by more severe social withdrawal. Therefore, the current findings might underrepresent mortgage victims' mental health.

\section{Conclusions}

Despite its limitations, this study helps confirm the profound psychological distress suffered by people who are in an eviction process. The risk of suicide is the "tip of an iceberg" that points to a diffused and profound experience of mental discomfort and helplessness associated with eviction, and this worsens over time. It also helps to identify some situations that aggravate this situation, such as the lack of social support and psychological and emotional help, and the menacing contact of the banks.

As concluded by Rojas and Sternberg [28] "the legitimacy of using evictions as a general preventive measure to promote [loan payback] needs to be viewed in relation to" the repercussions of evictions on mortgage victims' health.

\section{Abbreviations}

CJEU: Court of Justice of the European Union; ER: Emergency room; MINI: The Mini International Neuropsychiatric Interview; PTSD: Post-traumatic stress disorder; WHO: World Health Organization

\footnotetext{
Acknowledgments

We are especially thankful to all participant subjects, and to Antonio Redondo Aguado, from Stop Desahucios (Stop Evictions) Granada, for his support and assistance through the carrying out of the study. Also we thank C. Escudero, C Sánchez-Cantalejo, M. Teresa Alonzo, Alba Asensio, Rafael Beato, Laura Cabeza, Nicolás Cabib, Ana Maria Carrillo, Saray Cimadevilla, Alba Garrido, Miguel Angel Iñiguez, Miriam Ferrer, Laura Lopez, Marta Llergo, Laura Mas, M. Estefania Martinez, Nerea Martinez, Celia Moreno, Lisa Andrea Mazzoni, Ana Maria Perandres, Jose Olmedo, Sofía Orellana, Alejandra Ortega, Irene Pacheco, Jessica Perez, Elia Quesada, Maria Rodriquez, Joana Romero, Maria Rubin de Celix y Carolina Salvador, for their availability to conduct interviews. Finally, to the Andalusian School of Public Health and the Faculty of Psychology of the University of Granada for their support to this project.
}

\section{Authors' contributions}

IMR and ADC have coordinated the project, and both with JVC have designed it. IMR, HR, MCFS, JLMM and JVC have selected the psychological scales. IMR, ADC, and JBM developed and adapted the questionnaire used in the project. IMR, ADC, JVC, and MCFS have coordinated the fieldwork. MBS, JBM, HRO, JLMM, MCFS, IMR, and CE have organized and developed the fieldwork. IMR and ADC have analyzed the data. IMR and LM have prepared the first draft of the article. ADC is responsible for the final revisions. All the authors have participated in the edition and revision of the successive drafts of the article, and they are in agreement with the final version.

Funding

This research received no external funding

\section{Availability of data and materials}

The datasets used during the current study are available from the corresponding author on reasonable request.

\section{Ethics approval and consent to participate}

This study was approved by the Ethics Committee for Research of Granada, in Spain. At the beginning of the survey, an institutional letter was delivered to each selected subjects, presenting the study and other relevant information of the project, and written informed consent was obtained.

\section{Consent for publication}

'Not Applicable'.

\section{Competing interests}

The authors declare that they have no competing interests.

\section{Author details}

${ }^{1}$ CIBER Epidemiology and Public Health (CIBERESP), Instituto de Salud Carlos III, Madrid, Spain. ${ }^{2}$ Andalusian School of Public Health (EASP), Granada, Spain. ${ }^{3}$ Institute of Biomedicine Research (IBIS) Granada, Granada, Spain.

${ }^{4}$ Andalusian Observatory on Environment and Health (OSMAN), Granada, Spain. ${ }^{5}$ Mind, Brain, and Behavior Research Center (CIMCYC), University of Granada, Granada, Spain. ${ }^{6}$ Observatorio de Salud y Medio Ambiente de Andalucía, Escuela Andaluza de Salud Pública (EASP), Consejería de Salud y Familias de la Junta de Andalucia, Cuesta del Observatorio,4, 18080 Granada, Spain

Received: 24 April 2019 Accepted: 26 August 2019

Published online: 11 September 2019

\section{References}

1. Currie J, Tekin E. Is there a link between health and foreclosure? Am Econ J Econ Policy. 2015:7:63-94. https://doi.org/10.1257/pol.20120325.

2. Méndez Gutiérrez del Valle, R., Plaza Tabasco, J., 2018. Crisis inmobiliaria y desahucios hipotecarios en España: una perspectiva geográfica. Boletín la Asoc Geógrafos Españoles 99-127. doi: https://doi.org/10.21138/bage.2276

3. Bacigalupe A, Shahidi FV, Muntaner C, Martín U, Borrell C. Why is there so much controversy regarding the population health impact of the great recession? Reflections on three case studies. Int J Health Serv. 2016;46:5-35. https://doi.org/10.1177/0020731415611634.

4. Barr B, Taylor-Robinson D, Scott-Samuel A, McKee M, Stuckler D. Suicides associated with the 2008-10 economic recession in England: time trend analysis. BMJ. 2012;345:e5142. https://doi.org/10.1136/bmj.e5142.

5. Gili M, Roca M, Basu S, McKee M, Stuckler D. The mental health risks of economic crisis in Spain: evidence from primary care centres, 2006 and 2010. Eur J Pub Health. 2013;23:103-8. https://doi.org/10.1093/eurpub/ cks035.

6. Granizo JJ, Guallar E, Rodríguez-Artalejo F. Age-period-cohort analysis of suicide mortality rates in Spain, 1959-1991. Int J Epidemiol. 1996;25:814-20. https://doi.org/10.1093/ije/25.4.814.

7. Houle JN, Light MT. The home foreclosure crisis and rising suicide rates, 2005 to 2010. Am. J. Public Health. 2014;104:1073-9. https://doi.org/10.2105/ AJPH.2013.301774.

8. Osypuk TL, Caldwell CH, Platt RW, Misra DP. The consequences of foreclosure for depressive symptomatology. Ann Epidemiol. 2012;22:379-87. https://doi.org/10.1016/j.annepidem.2012.04.012. 
9. Reeves A, Stuckler D, McKee M, Gunnell D, Chang S-S, Basu S. Increase in state suicide rates in the USA during economic recession. Lancet. 2012;380: 1813-4. https://doi.org/10.1016/S0140-6736(12)61910-2.

10. Ruiz-Perez I, Rodriguez-Barranco M, Rojas-Garcia A, Mendoza-Garcia O. Economic crisis and suicides in Spain. Socio-demographic and regional variability. Eur J Heal Econ. 2017;18:313-20. https://doi.org/10.1007/s10198016-0774-5.

11. Salmerón D, Cirera L, Ballesta M, Navarro-Mateu F. Time trends and geographical variations in mortality due to suicide and causes of undetermined intent in Spain, 1991-2008. J Public Health. 2013;35:237-45. https://doi.org/10.1093/pubmed/fds103.

12. Stuckler D, Basu S, Suhrcke M, Coutts A, McKee M. Effects of the 2008 recession on health: a first look at European data. Lancet. 2011;378:124-5. https://doi.org/10.1016/S0140-6736(11)61079-9.

13. Zivin K, Paczkowski M, Galea S. Economic downturns and population mental health: research findings, gaps, challenges and priorities. Psychol Med. 2011;41:1343-8. https://doi.org/10.1017/S003329171000173X.

14. Jones RW, Pridemore WA. The U.S. housing crisis and suicide rates: an examination of total-, sex-, and race-specific suicide rates. Hous Stud. 2016; 31:173-89. https://doi.org/10.1080/02673037.2015.1070795.

15. Novoa A, Bosch J, Dìaz F, Malmusi D, Darnell M, Trilla C. El impacto de la crisis en la relación entre vivienda y salud Políticas de buenas prácticas para reducir las desigualdades en salud asociadas con las condiciones de vivienda. Gac Sanit. 2014;28:44-50. https://doi.org/10.1016/j.gaceta.2014.02.018.

16. Vasquez-Vera H, Palencia L, Magna I, Mena C, Neira J, Borrell C. The threat of home eviction and its effects on health through the equity lens: a systematic review. Soc Sci Med. 2017;175:199-208. https://doi.org/10.1016/j. socscimed.2017.01.010.

17. Parreño-Castellano J, Domínguez-Mujica J, Armengol-Martín M, Pérez García T, Boldú Hernández J. Foreclosures and evictions in Las Palmas de gran Canaria during the economic crisis and post-crisis period in Spain. Urban Sci. 2018;2:109. https://doi.org/10.3390/urbansci2040109.

18. Houle JN, Light MT. The harder they fall? Sex and race/ethnic specific suicide rates in the U.S. foreclosure crisis. Soc Sci Med. 2017;180:114-24. https://doi.org/10.1016/j.socscimed.2017.03.033.

19. Keene DE, Cowan SK, Baker AC. "When you're in a crisis like that, you don't want people to know": mortgage strain, stigma, and mental health. Am. J. Public Health. 2015:105:1008-12. https://doi.org/10.2105/AJPH.2014.302400.

20. Nettleton S, Burrows R. When a capital investment becomes an emotional loss: the health consequences of the experience of mortgage possession in England. Hous Stud. 2000;15:463-79. https://doi.org/10.1080/0267303005 0009285.

21. Ross LM, Squires GD. The personal costs of subprime lending and the foreclosure crisis: a matter of trust, insecurity, and institutional deception. Soc Sci Q. 2011;92:140-63. https://doi.org/10.1111/j.1540-6237.2011.00761.x.

22. Bolívar Muñoz J, Bernal Solano M, Mateo Rodríguez I, Daponte Codina A Escudero Espinosa C, Sánchez Cantalejo C, González Usera I, Robles Ortega H, Mata Martín JL, Fernández Santaella MC, Vila CJ. The health of adults undergoing an eviction process. Gac Sanit. 2016:30(1):4-10.

23. Burgard SA, Seefeldt KS, Zelner S. Housing instability and health: findings from the Michigan recession and recovery study. Soc Sci Med. 2012;75: 2215-24. https://doi.org/10.1016/j.socscimed.2012.08.020.

24. Bachmann S. Epidemiology of suicide and the psychiatric perspective. Int. J. Environ. Res. Public Health. 2018;15:1-23. https://doi.org/10.3390/ijerph15 071425.

25. Wasserman D, Wasserman C. Oxford textbook of suicidology and suicide prevention. Oxford: Oxford University Press; 2009.

26. World Health Organization. Practice manual for establishing and maintaining surveillance systems for suicide attempts and self-harm. Geneva; 2016. https://doi.org/10.1063/1.2364135.

27. Fowler KA, Gladden RM, Vagi KJ, Barnes J, Frazier L. Increase in suicides associated with home eviction and foreclosure during the US housing crisis: findings from 16 national violent death reporting system states, 2005-2010. Am J Public Health. 2015;105:311-6. https://doi.org/10.2105/AJPH.2014.301945.

28. Rojas Y, Stenberg SÅ. Evictions and suicide: a follow-up study of almost 22 000 Swedish households in the wake of the global financial crisis. J Epidemiol Community Health. 2016;70:409-13. https://doi.org/10.1136/ jech-2015-206419.

29. Serby MJ, Brody MD, Shetal Amin BA, Yanowitch MD. Eviction as a risk factor for suicide. Psychiatr. Serv. 57, 273-b. 2006. https://doi.org/10.1176/ appi.ps.57.2.273-b.
30. Bernal-Solano M, Bolívar-Muñoz J, Mateo-Rodríguez I, et al. Associations between Home Foreclosure and Health Outcomes in a Spanish City. Int J Environ Res Public Health. 2019;16(6):981. Published 2019 Mar 19. https:// doi.org/10.3390/ijerph16060981.

31. McKee M, Reeves A, Clair A, Stuckler D. Living on the edge: precariousness and why it matters for health. Arch Public Heal. 2017;75:1-10. https://doi. org/10.1186/s13690-017-0183-y.

32. Alvarez-Galvez J, Salinas-Perez JA, Rodero-Cosano ML, Salvador-Carulla L. Methodological barriers to studying the association between the economic crisis and suicide in Spain. BMC Public Health. 2017;17:1-10. https://doi. org/10.1186/s12889-017-4702-0.

33. de León AC, Rodríguez IM, Gannar F, Pedrero García AJ, González DA, del Cristo Rodríguez Pérez M, Díaz BB, Sánchez JJA, Aguirre-Jaime A. Austerity policies and mortality in Spain after the financial crisis of 2008. Am J Public Health. 2018;108:1091-8. https://doi.org/10.2105/AJPH.2018.304346.

34. Karanikolos M, Mladovsky P, Cylus J, Thomson S, Basu S, Stuckler D, MacKenbach JP, McKee M. Financial crisis, austerity, and health in Europe. Lancet. 2013;381:1323-31. https://doi.org/10.1016/S0140-6736(13)60102-6.

35. Stuckler D, Basu S, Suhrcke M, Coutts A, McKee M. The public health effect of economic crises and alternative policy responses in Europe: an empirical analysis. Lancet. 2009:374:315-23. https://doi.org/10.1016/S0140-6736 (09)61124-7.

36. European Commission, OECD, Storper M, Scott AJ. The urban and regional dimension of the crisis. Luxembourg: Publications Office of the European Union; 2013. https://doi.org/10.2776/74866.

37. Buendía L. A perfect storm in a sunny economy: a political economy approach to the crisis in Spain. Socio-Economic Rev. 2018;0:1-20. https:// doi.org/10.1093/ser/mwy021.

38. Daponte Codina A, Mateo Rodríguez I, Vásquez-Vera H. Los desahucios y la salud, se necesita una respuesta desde la salud pública en España. Gac Sanit. 2016;30:239-41. https://doi.org/10.1016/j.gaceta.2016.03.012.

39. Eurostat. Unemployment Statistics. At: https://ec.europa.eu/eurostat/ statistics-explained/index.php/Unemployment_statistics. Accessed Mar 2019.

40. Ayala-Nunes $L$, Jiménez $L$, Jesus $S$, Hidalgo V. Social support, Economic Hardship and Psychological Distress in Spanish and Portuguese At-Risk Families. J Child Fam Stud. 2018;27:176-86. https://doi.org/10.1007/s10826017-0863-9.

41. Court of Justice of the European Union/CJEU (CJEU, 14 March 2013, Case C415/11; case Gutierrez Naranjo, Joined Cases C-154/15, C-307/15 and C-308/ 15, EU:C:2016:980; case Banco Primus, Case C-421/14, EU:C:2017:60). At: https://curia.europa.eu/jcms/jcms/j_6/es/. Accessed Feb 2019.

42. Álvarez de Andrés E, Zapata Campos MJ, Zapata P. Stop the evictions! The diffusion of networked social movements and the emergence of a hybrid space: The case of the Spanish Mortgage Victims Group. Habitat Int. 2015: 46:252-9. https://doi.org/10.1016/j.habitatint.2014.10.002.

43. Nock MK, Borges G, Bromet EJ, Cha CB, Kessler RC, Lee S. Suicide and suicidal behavior. Epidemiol Rev. 2008;30(1):133-54.

44. Li Z, Page A, Martin G, Taylor R. Attributable risk of psychiatric and socioeconomic factors for suicide from individual-level, population-based studies: a systematic review. Soc Sci Med. 2011;72(4):608-16.

45. Mejías-Martín Y, Martí-García C, Rodríguez-Mejías C, Valencia-Quintero JP, García-Caro MP, de Dios Luna J. Suicide attempts in Spain according to prehospital healthcare emergency records. PLoS One. 2018;13:1-13. https:// doi.org/10.1371/journal.pone.0195370.

46. Robles-Ortega H, Guerra P, González-Usera I, Mata-Martín JL, FernándezSantaella MC, Vila J, Bolívar-Muñoz J, Bernal-Solano M, Mateo-Rodríguez I, Daponte-Codina A. Post-traumatic stress disorder symptomatology in people affected by home eviction in Spain. Span J Psychol. 2017;20:E57

47. Heckathorn DD. Respondent-driven sampling: a new approach to the study of hidden populations. Soc Probl. 1997;44:174-99. https://doi.org/10.2307/3 096941.

48. Sordo L, Pérez-Vicente S, Rodríguez Del Águila MM, Bravo MJ. Muestreo dirigido por los participantes Para el estudio de poblaciones de difícil acceso. Med Clin (Barc). 2013;140:83-7. https://doi.org/10.1016/j.medcli.2012.07.017.

49. Lecrubier $Y$, Sheehan DV, Weiller E, Amorim P, Bonora I, Sheehan KH, Janavs J, Dunbar GC. The MINI international neuropsychiatric interview (MINI). A short diagnostic structured interview: Reliability and validity according to the CIDI. Eur Psychiatry. 1997;12:224-31. https://doi.org/10.1016/S0924-933 8(97)83296-8.

50. Domingo-Salvany A, Bacigalupe A, Carrasco JM, Espelt A, Ferrando J, Borrell C. Propuestas de clase social neoweberiana y neomarxista a partir de la 
Clasificación Nacional de Ocupaciones 2011 [proposals for social class classification based on the Spanish National Classification of occupations 2011 using neo-Weberian and neo-Marxist]. Gac Sanit. 2013;27:263-72. https://doi.org/10.1016/j.gaceta.2012.12.009.

51. Broadhead W, Gehlbach S, Degruy F, Kaplan B. The Duke-UNK functional social support questionnaire: measurement of social support in family medicine patients. Med Care. 1988;26:709-23.

52. Huerta-Ramírez R. Conducta suicida en población general adulta española. Inf Psiquiátricas. 2017;227:25-42.

53. Bernal M, Haro JM, Bernert S, Brugha T, de Graaf R, Bruffaerts R, Lépine JP, de Girolamo G, Vilagut G, Gasquet I, Torres JV, Kovess V, Heider D, Neeleman J, Kessler R, Alonso J. Risk factors for suicidality in Europe: results from the ESEMED study. J Affect Disord. 2007;101:27-34. https://doi.org/10.1 016/j.jad.2006.09.018.

54. Arias-de la Torre, J., Molina, A.J., Fernández-Villa, T., Artazcoz, L., Martín, V., 2017. Mental health, family roles and employment status inside and outside the household in Spain. Gac Sanit. do: https://doi.org/10.1016/j.gaceta.201 7.11.005.

55. Masana L. Long-term informal care in Spain: challenges, views and solutions. Salud Colect. 2017;13(2):337-52. https://doi.org/10.18294/sc.201 7.1237.

56. INE, 2014a. Instituto Nacional de Estadística. Madrid: National Health Survey [WWW Document].

57. Uchino BN, Cacioppo JT, Kiecolt-Glaser JK. The relationship between social support and physiological processes. Psychol Bull. 1996;1 19:488-531. https:// doi.org/10.1037/0033-2909.119.3.488.

58. Chang, E.C., Sanna, L.J., Hirsch, Jameson, K., Jeglic, E.L., 2010. Loneliness and negative life events as predictors of hopelessness and suicidal behaviors in Hispanics: evidence for a diathesis-stress model. J Clin Psychol 62, 12421253. doi: https://doi.org/10.1002/jclp.

59. Conroy RW, Smith K. Family loss and hospital suicide. Suicide Life- Threat Behav. 1983;13:179-94.

60. Shaw R, Graham N, Mackay D, Ward J, Pearsall R, Smith D. Loneliness, living arrangements and emotional support as predictors of suicidality: a 7 year follow-up of the Uk biobank cohort. J Epidemiol Community Health. 2018: 30-1. https://doi.org/10.1136/jech-2018-SSMabstracts.62.

61. Stravynski A, Boyer R. Loneliness in relation to suicide ideation and Parasuicide: a population-wide study. Suicide Life-Threatening Behav. 2001; 31:32-40. https://doi.org/10.1521/suli.31.1.32.21312.

62. Miret M, Caballero FF, Huerta-Ramírez R, Moneta MV, Olaya B, Chatterij S, Haro JM, Ayuso-Mateos JL. Factors associated with suicidal ideation and attempts in Spain for different age groups. Prevalence before and after the onset of the economic crisis. J Affect Disord. 2014;163:1-9. https://doi.org/1 0.1016/j.jad.2014.03.045.

63. Márquez-Calderón S, Villegas-Portero R, Gosalbes Soler V, Martínez-Pecino F. Promoción de la salud y prevención en tiempos de crisis: El rol del sector sanitario. Informe SESPAS 2014. Gac Sanit. 2014;28:116-23. https://doi.org/1 0.1016/j.gaceta.2014.01.012.

64. Martin-Carrasco M, Evans-Lacko S, Dom G, Christodoulou NG, Samochowiec J, González-Fraile E, Bienkowski P, Gómez-Beneyto M, Dos Santos MJH, Wasserman D. EPA guidance on mental health and economic crises in Europe. Eur Arch Psychiatry Clin Neurosci. 2016;266:89-124. https://doi.org/1 0.1007/s00406-016-0681-X.

65. Segura Benedicto, A., 2018. El sistema sanitario, la atención primaria y la salud. Health system, primary care and public health. Atención Primaria 50, 388-389. do: https://doi.org/10.1016/j.aprim.2018.01.001.

66. Armas CS, García MH, Cofiño R. ¿De qué hablamos cuando hablamos de «salud comunitaria»? Informe SESPAS 2018. Gac Sanit. 2018:5-12. https://doi. org/10.1016/j.apsusc.2017.01.140.

67. Gili M, García Campayo J, Roca M. Crisis económica y salud mental. Informe SESPAS 2014. Gac Sanit. 2014;28:104-8. https://doi.org/10.1016/j.gaceta.2014. 02.005 .

68. Buitrago Ramírez F, Misol RC, Bentata LC, Fernández Alonso MDC, Campayo JG, Franco CM, Tizón García JL. Recomendaciones Para la prevención de los trastornos de la salud mental en atención primaria. Aten Primaria. 2016;48: 77-97. https://doi.org/10.1016/S0212-6567(16)30189-5.

69. World Health Organization. The World health report : 2001 : Mental health : new understanding, new hope. World Health Organization; 2001.

70. Saxena S, Sharan P, Garrido-Cumbrera M, Saraceno B. World Health Organization's mental health atlas 2005: implications for policy development. World Psychiatry. 2006;5:180-4.
71. Mann J. Health and human rights: if not now, when? Health hum. Rights. 1997;2:113-20. https://doi.org/10.2307/4065162.

72. Gruskin S, Mills EJ, Tarantola D. History, principles, and practice of health and human rights. Lancet. 2007;370:449-55. https://doi.org/10.1016/S0140-6 736(07)61200-8.

73. Pickett KE, Wilkinson RG. Income inequality and health: a causal review. Soc Sci Med. 2015;128:316-26. https://doi.org/10.1016/j.socscimed.2014.12.031.

74. OECD. Crisis squeezes income and puts pressure on inequality and poverty: results from the OECD Income Distribution Database. Paris: Organization for Economic Cooperation and Development; 2013.

75. De Vogli R. The financial crisis, health and health inequalities in Europe: the need for regulations, redistribution and social protection. Int J Equity Health. 2014;13:58.

76. Fernández Ruiz-Galvez E. Rebus sic stantibus y crisis económica. Orden público económico versus especulación Anu Filos del Derecho Xxxiii; 2017. p. 63-98.

77. Sánchez Ruiz de Valdivia I, Olmedo Cardenete MD. Desahucios y ejecuciones hipotecarias: un drama social y un problema legal. Valencia: Tirant Lo Blanch; 2014.

78. Nasarre Aznar S. Cuestionando algunos mitos del acceso a la vivienda en España, en perspectiva europea. Cuad Relac Laborales. 2017;35:43-69. https://doi.org/10.5209/CRLA.54983.

79. Oliva Moreno J, González López-Varcárcel B, Barber P, Peña Longobardo LM Urbanos Garrido R, Zozaya González N. Crisis económica y salud en España. Madrid: Ministerio De Sanidad, Consumo Y Bienestar Social; 2018.

80. Houle JN. Mental health in the foreclosure crisis. Soc Sci Med. 2014:118:1-8. https://doi.org/10.1016/j.socscimed.2014.07.054.

81. Kentikelenis A, Karanikolos M, Reeves A, McKee M, Stuckler D. Greece's health crisis: from austerity to denialism. Lancet. 2014;383:748-53. https:// doi.org/10.1016/S0140-6736(13)62291-6.

82. Rachiotis G, Stuckler D, McKee M, Hadjichristodoulou C. What has happened to suicides during the Greek economic crisis? Findings from an ecological study of suicides and their determinants (2003-2012). BMJ Open. 2015;5:1-6. https://doi.org/10.1136/bmjopen-2014-007295.

83. Reeves, A., McKee, M., Stuckler, D., 2014. Economic suicides in the great recession in Europe and North America. Br J Psychiatry 205, 246-247. do: https://doi.org/10.1192/bjp.bp.114.144766.

84. Rafael Arredondo Quijada, María de las Olas Palma García 2013. Aproximación a la realidad de los desahucios: perfil y características de las familias en proceso de desahucios en la ciudad de Málaga. Altern Cuad Trab Soc doi: https://doi.org/10.14198/altern2013.20.07.

85. Observatorio DESC y Plataforma de Afectados por la Hipoteca. Emergencia habitacional en Cataluña. Impacto de la crisis hipotecaria en el derecho a la salud y los derechos de la infancia. Observatorio DESC y Plataforma de Afectados por la Hipoteca. Barcelona; 2015.

86. General Council of the Judiciary (CGPJ). 2016. Available online: http://www. poderjudicial.es/cgpj/es/Temas/Estadistica-Judicial/Informes-estadisticosperiodicos/Datos-sobre-el-efecto-de-la-crisis-en-losorganos-judiciales\%2D\%2 D-Datos-desde-2007-hasta-cuarto-trimestre-de-2015. Accessed Dec 2016.

87. Kingsley GT, Smith RE, Price D. The impacts of foreclosures on families and communities. Washington, DC: The Urban Institute; 2009.

88. Mykyta, L., 2018. Housing crisis, hardship and safety net support: examining the effects of foreclosure on households and families. Hous Stud 1-22. do: https://doi.org/10.1080/02673037.2018.1487040.

89. Lopez-Castroman J, Blasco-Fontecilla H, Courtet P, Baca-Garcia E, Oquendo MA. Are we studying the right populations to understand suicide? World Psychiatry. 2015;14:368-9. https://doi.org/10.1002/wps.20261.

\section{Publisher's Note}

Springer Nature remains neutral with regard to jurisdictional claims in published maps and institutional affiliations. 